\title{
Clinical Assessment and Management of Dapsone-resistant Leprosy for the Field Worker
}

\author{
GRACE WARREN* \\ The Leprosy Mission, c/o Christian Hospital, Manorom, Thailand
}

"Doctor, would you please look at this patient and advise me what to do. She has chronic Erythema Nodosum Leprosum (ENL) and does not respond to corticosteroids", asked the paramedical worker. The writer turned to see a young woman covered with hundreds of small to medium sized infiltrated lesions. Her history? She had first been diagnosed, as a teenager, some 10 years ago and started on dapsone. The treatment had been supervised by the paramedical worker most of the time without reference to a doctor. As the woman had had repeated bouts of ENL over the years her treatment had been rather irregular as the dapsone was usually stopped either by the paramedical worker or the patient whenever the ENL became severe. In the 1960s she had never received more than $100 \mathrm{mg}$ twice weekly, and often had received much less, but her Bacillary Index (BI) had fallen until it was almost zero in 1970. At this time the dose of dapsone had been increased to $50 \mathrm{mg}$ daily and maintained at that level. She had failed to attend clinic in 1975 and on careful questioning we extracted the information that there were already some new lesions appearing at her last attendance at clinic in October 1974. It was the persistence of these lesions and the increase in their number that had brought her back to clinic in March 1976. Because of the number of lesions, in a previously clear skin, the paramedical worker had assumed that she was having ENL and had given her corticosteroids and antihistamines. When she seemed to be getting worse rather than better he brought her to see the writer on my next visit, in June 1976. On careful examination it was obvious that these were new skin lesions and not ENL and her skin smear showed many bacilli, of which a fairly high proportion were solid in form. This then was a case of definite relapse, not of ENL, and the opportunity was taken of pointing out the difference in appearance. But the problem now was "Are the organisms still sensitive to dapsone or are they resistant?" As the lesions had appeared before she stopped dapsone it suggested resistance, especially with the history of low irregular dosage. But how to check her out with a poor laboratory cover?

The next patient had a similar problem. Bacillary resistance to dapsone is becoming a real problem in the field especially where much of the work has to be left to those who have been only partially trained and not recently updated. Many of them are not yet aware of the possibilities of dapsone resistance.

\footnotetext{
* Advisor in Leprosy and Reconstructive Surgery for the Leprosy Mission in Asia.
} Received for publication 10 February, 1977. 
As the writer travels through Asia she is repeatedly confronted with similar problems-patients who have been on treatment for some time and now are not doing as well as expected. Yes, a relatively high proportion of patients that I see are called specially because they are not responding adequately to their treatment, but even taking that into consideration, the numbers developing resistance, and their prognosis, if not adequately treated, is alarming. Let me first discuss the reasons why we are now having to combat so much resistance "on the field".

Dapsone was first used for the treatment of leprosy in the 1940s. Due to the inability to culture the organism the effective dosage had to be determined by trial and error. Initially patients received a high dosage which reportedly produced undesirable side effects, so that the dose was gradually reduced. In the 1950s the dose was usually between 400 and $800 \mathrm{mg}$ weekly given in one or 2 doses, of ten by injection in institutions. In 1960, after 10-15 years of intensive usage there was no real evidence suggesting that resistance to dapsone occurred, so in the early 1960s workers were informed that there was no need to give dual therapy as was given in tuberculosis.

With the advent of the mouse foot-pad culture techniques the possibility of controlled drug trials became a reality and the treatment of leprosy came under more detailed and systematic investigation. Workers were informed that lower doses of dapsone were effective and produced less undesirable side effects. In the late 1960s doses as low as $1 \mathrm{mg}$ per day were shown to be effective as an initial treatment, but at the same time they were not recommended, as warnings of the development of resistance to dapsone were also appearing.

Field workers were in a quandary. Many had reduced the dosage of dapsone in good faith so that patients were receiving doses of $10,25,50$ or $100 \mathrm{mg}$ twice weekly. On this they seemed to do well, the skin lesions healed and there seemed to be few adverse side effects. But, every now and again, one would find a patient who just did not seem to respond adequately to dapsone, either in large or small doses. Why was this?

The writer remembers 2 such cases. One was initially seen in 1966. He was a man of 60 years who had been treated with all the sulphones for 20 years. He had had dapsone, sulphetrone, diasone and promin, but they had been stopped and started as he frequently had ENL. When he was first seen in Hong Kong to which he had recently immigrated he appeared to be of the florid lepromatous type with a BI of 5.5 and a Morphological Index (MI) of 60\% solid rods. Dapsone by injection in large doses for 6 months made little difference to the BI and MI and he had repeated ENL. After commencing Vadrine (which was the only drug available then that he had not already had) he did show some slow improvement. Eventually he did well on clofazimine when it became available in late 1967.

Another patient in Korea had received promin injections 3 times weekly for about 15 years. He presented with many new lesions-could this be resistance? He was adamant that he had not been irregular with his therapy. Yet at that time workers were being told "resistance to dapsone does not occur". All through the 1960s patients like this were being found. By 1970 the writer had some 15 or 20 under her care in Hong Kong alone. They were mostly patients who had been under treatment for a long time or who had been irregular with their treatment. Could one always blame the patient for irregularity?

Soon after dapsone became accepted as a standard treatment for leprosy it was observed that many patients under treatment developed severe ENL. It was assumed that the dapsone produced the ENL, though on careful observation one 
realizes that patients get ENL without taking dapsone. Nevertheless the custom arose of stopping dapsone whenever reaction became severe, and patients learnt to stop it themselves. This meant that many patients in the LL, LI and LB groups were receiving irregular treatment because of recurrent ENL. But was this the only reason for their irregularity?

The usual instructions were to divide the weekly dose into 2 parts to be taken on 2 days. It is hard to regularly remember to do something twice a week, and one missed dose meant half the dose for that week was missed. Some workers realized that and daily dosage was instituted in some centres in the early 1960s. In some institutions the taking of dapsone was "supervised"-well the medicine was handed out and meant to be swallowed in front of the staff member. But, the number of "sucked" dapsone tablets available on the "black market" was mute evidence to the unreliability of this system.

Bearing this in mind dapsone was often given by injection, especially to relapsed patients, with very good initial effect. Was it a problem of malabsorption? Sometimes dapsone tablets are stored for long periods and may become very hard. Sometimes in the manufacture they are compressed so hard that they can pass through the gut unaltered. It is easy to test solubility by dropping one into a glass of water but that does not of necessity say anything about the absorption of the drug.

So through the 1960s more clinical evidence was collecting to be confirmed by laboratory studies with the mouse foot-pad. Why shouldn't the bacilli become resistant to dapsone? Other bacilli become resistant to drugs, and in a much shorter time that $M$. leprae had needed to show resistance to dapsone. Yes now it is accepted, the bacilli were much smarter than we thought they were, not only can they become resistant to dapsone but to other antileprosy drugs also. They have also revealed themselves as being able to remain alive and viable, for years, in the presence of a concentration of drug that should be enough to eliminate the infection. These bacilli are called persisters and may complicate the diagnosis of dapsone resistance, as they resume multiplication when the dapsone level falls and produce relapse that is fully sensitive to dapsone.

So workers cannot assume that every relapsed patient has dapsone resistant leprosy. As most centres do not have the facilities for mouse foot-pad tests a clinical test for resistance is essential. The writer started using that in the late 1960s in Hong Kong and on a few occasions was able to check results with mouse foot-pad inoculation. In each patient so tested dapsone resistance was confirmed in the mouse, and usually these patients showed resistance to thiambutasone as well as it had often been used for patients who did not show adequate progress on dapsone.

This then is the problem that we now face. Let us look at it more systematically.

\section{Who to Suspect}

Workers must learn to think of dapsone resistance as a possibility in any leprosy patient who had been multibacilliferous and has received dapsone for a fairly long period of time and is now showing signs of relapse. The possibilities of resistance increase with:

1. The nearness to the lepromatous end of the immunological spectrum (resistance has so far only been proved in patients who were initially LL, LI or LB in type). 
2. The longer the period of dapsone medication.

3. The smaller the dose of dapsone that has been used.

4. The irregularity of the dapsone medication.

5. The consistent use of monotherapy.

The typical patient is an LL type patient who has been on treatment for 10-15 years with dapsone alone in relatively low doses that have been interrupted because of reaction (or other causes) or lack of co-operation. However resistance has been proved in patients under treatment for only 4-5 years and in those who have received high doses of dapsone regularly for 20 years.

\section{What Does It Look Like?}

The lesions of dapsone-resistant leprosy do not really differ in appearance from the lesions of ordinary dapsone-sensitive relapsed leprosy. It may be possible to see the old healed lesions behind the new ones which are usually reddish (in the lighter skins, bronzed in darker skins) papules or macules. They may become heavily infiltrated rather more rapidly than normally expected. There may be plaques of infiltration, or with time nodules may develop which may become very gross. It has been stated that one can recognize dapsone resistance by an umbilication of the papules and nodules, but the writer has not found this so. Sometimes the new lesions appear to be just a flat, non-irritant, persistent rash on the forearms that can be mistaken for a drug eruption. The patient does not feel ill, he has no fever or general symptoms such as one may get in lepra reaction-though of course he may have lepra reaction as well as relapse. On skin smear (or biopsy) in relapsed leprosy the lesions will be found to be teeming with acidfast bacilli (AFB). The most productive site will be a small fleshy papule that has newly developed. If resistance is suspected and the first smear is negative or less than suspected it should be repeated each 2 weeks until the lesions subside or the diagnosis is determined.

Relapsed leprosy covers any situation in which new active lesions appear in a patient already responded to treatment. The relapse may be due to dapsone resistance, failure to take dapsone (or other antileprotic drug) in adequate dosage, or to persister organisms. It is important that the cause of the relapse be determined before there is any change in specific drug therapy.

\section{Differential Diagnosis}

The lesions of relapsed leprosy are often mistaken for ENL especially by paramedical workers who have been taught to watch for ENL and not to suspect resistance. Why shouldn't they think they are ENL? ENL is common; teaching may have neglected resistance; both show new reddish lesions in a patient who is under treatment. But it should not be difficult to distinguish ENL from the lesions of relapse. Table 1 should help paramedical and other field workers who are not yet familiar with the difference.

If the patient has ever had ENL before he will usually realize that the lesions of relapse are not the same. It may be helpful to count the lesions on a defined area (such as an arm) and observe if they fluctuate over a period of weeks. With ENL the number will fluctuate from day to day but in relapse they will increase in number and the lesions may get larger. 
TABLE 1

Differentiation between ENL and relapsed leprosy

\begin{tabular}{|c|c|}
\hline ENL & Relapse \\
\hline Crops of lesions that come and go & Lesions that persist \\
\hline Lesions may be tender & Lesions are not tender \\
\hline Lesions may ulcerate & Lesions usually do not ulcerate \\
\hline $\begin{array}{l}\text { On pressure with a glass slide the lesions } \\
\text { will disappear, but sometimes there may } \\
\text { remain a dark spot in the centre if } \\
\text { thrombosis has occurred }\end{array}$ & $\begin{array}{l}\text { There is true infiltration and associated } \\
\text { erythema which do not completely } \\
\text { disappear on pressure }\end{array}$ \\
\hline Lesions are not really infiltrated & $\begin{array}{l}\text { True infiltration may go on to papules, } \\
\text { nodules and plaques }\end{array}$ \\
\hline $\begin{array}{l}\text { Systemic symptoms of pain, fever and } \\
\text { malaise may dominate the picture }\end{array}$ & There may be no systemic symptoms \\
\hline Urine may show $\mathrm{RBC}$, and albumin & No specific urinary changes \\
\hline May be acute anaemia in some races & No haemoglobin changes \\
\hline $\begin{array}{l}\text { Lesions usually subside with } \\
\text { corticosteroids }\end{array}$ & $\begin{array}{l}\text { Lesions get worse if corticosteroids are given } \\
\text { without antileprotics }\end{array}$ \\
\hline
\end{tabular}

\section{Investigation}

(a) Check the history as carefully as possible.

(b) Is the patient reliable-did he really take his dapsone as he says he did. Is there anyone who can verify his story?

(c) Take skin smears and check BI and MI at 6 sites and also take nasal swabs. It is essential that good laboratory coverage be available whenever dapsone resistance is being investigated.

(d) Tests for resistance.

(i) Laboratory. If possible take a biopsy from a site with a high BI and MI for mouse foot-pad investigation before giving any new specific medication.

(ii) Clinical testing in the absence of mouse foot-pad testing. This will be the only method of testing for most of the field workers.

\section{Methodology of Clinical Testing}

Carefully list the actual sites from which the skin smears were taken, and the individual results of $\mathrm{BI}$ and $\mathrm{MI}$ readings. Results from different sites can vary very much in the same patient and it is best to be able to follow the progress in a specific site.

Give $100 \mathrm{mg}$ dapsone daily-preferably under proper supervision (check that it is swallowed) or give by injection.

Repeat the smears from the same sites every 2 weeks.

If the MI falls consistently we can assume that the bacilli are still sensitive to dapsone. It may take 6-9 months for the MI to reach $0 \%$ solids in a badly relapsed patient who is still dapsone sensitive, and the BI may not fall significantly until the $\mathrm{MI}$ is $0 \%$ (observed in Chinese patients receiving intramuscular dapsone). So we do need to observe closely for a prolonged period of time. Smears should be done each 2 weeks for 4-6 times and then every 2-3 months for several years in any person who has been suspected of being dapsone 
resistant but shows clinical response to higher dapsone dosage. After an initial period of improvement in both BI and MI the smears may appear to remain stationary for months or even years and then there may be a sudden rise in the bacterial counts, which is usually accompanied by new lesions again. This would suggest that there has been a partial resistance to dapsone which has now become a complete resistance.

It is important, when no mouse foot-pads are available to make a definite decision regarding dapsone resistance as this decision may influence the whole of the patient's future life. It may be difficult but at some stage the decision must be made and the patient's chart endorsed accordingly and his treatment adjusted. If the MI has not fallen by $50 \%$ in the first 6 weeks on full dapsone dosage it is best to assume that the bacilli are dapsone resistant and commence alternative therapy as soon as possible.

\section{Management of Dapsone-resistant Leprosy}

First: endorse the patient's chart so that it cannot easily be missed.

Second: explain to the patient something of what has happened and of the severity of the situation and of the necessity of his being regular with therapy in the future if he is to get well and to stay well.

The patient who is dapsone resistant should never be given dapsone as the sole drug for treating his leprosy again. Theoretically it may still be of help in the patient who is partially resistant to dapsone, or in the prevention of a second infection with a dapsone-sensitive organism, but in both of these situations the organisms should be dealt with by the alternate drug given which is usually clofazimine. With our present drugs clofazimine is the only drug available for long term treatment of the patient with dapsone-resistant leprosy. As clofazimine pigments the skin it is essential that the patient realises the situation and is prepared to accept this pigmentation for life. If he is not convinced that clofazimine is essential he may stop it himself as soon as he looks better and resume dapsone with the result that he relapses again.

When it is possible to inoculate mouse foot-pads it is practical to give clofazimine as soon as the biopsy material is taken. If Rifampycin can be given also the patient will become non-infectious within 2 weeks and this is of obvious advantage when we are seeking to stop the spread of dapsone-resistant organisms. Once foot-pad inoculation is set up we can eventually determine the possibility of giving dapsone again later, though the patient may not readily accept the verdict that he is dapsone resistant, once he looks and feels better. On the other hand is the time taken to convince one patient that he is dapsone resistant, providing an unnecessarily long period during which dapsone-resistant organisms can be disseminated?

There are many different regimes for treating dapsone-resistant leprosy but it would appear that the use of Rifampycin $600 \mathrm{mg}$ daily with clofazimine $100 \mathrm{mg}$ daily for 2 weeks followed by clofazimine $100 \mathrm{mg}$ daily for the first 6 months has much to recommend it. At the end of 6 months the clofazimine can be reduced to alternate days for life. Unfortunately this means that the patient, after the initial 2 weeks is once again on monotherapy. For this reason workers are trying combinations of second line drugs in an attempt to reduce the incidence of further resistance but results of these trials will not be available for many years. 


\section{What Should Be Our Response to This Problem?}

Now that we know that dapsone resistance is a definite entity and that it also means that there is resistance to all the related sulphones and sulphonamides it is important that we modify our thinking in relation to those persons who are infected with $M$. leprae. How should we do this?

(1) Higher dosage of dapsone should be given as soon as possible, especially in patients with low resistance forms of leprosy (LL, LI \& LB types). At least $1 \mathrm{mg} / \mathrm{kg} / \mathrm{day}$ should be given within 6 months of the commencement of therapy and maintained for as long as dapsone is given. Daily therapy is preferable to twice weekly but second daily medication is probably acceptable. The writer is one who cannot agree that every patient should commence with $100 \mathrm{mg}$ daily. She has seen too many tragedies that have seemed to result from this form of therapy, but she does agree that very small doses should not be given even for short periods, unless a second drug is being used at the same time. This may happen when a patient needs to be desensitized to dapsone allergy and is receiving clofazimine during the desensitization. The use of smaller than maximal doses of dapsone may also be justified when it is being given as part of dual therapy in a patient who does not seem to tolerate a full dose of any antileprotic drug.

Regular therapy in adequate dosage is the key to the prevention of dapsone resistance. While we do all in our power to encourage the newly diagnosed patient to take his treatment regularly there comes a time when a different attitude may be wise. If a patient after many years' treatment is skin smear negative and becomes irregular in taking his dapsone it may be better to stop giving him dapsone. Of course the situation should be explained to him and he should be told that there is a chance of relapse, and that if new lesions appear he should return quickly for more treatment. If he has been irregular the chance of dapsone resistance is much greater, but if he has not been taking dapsone for some years he is more likely to be dapsone sensitive at relapse, and to take treatment regularly again, at least for some time. If we give dapsone irregularly not only are we encouraging resistance to occur but he may come to feel the treatment is no good and delay unduly when new lesions do occur.

(2) Wherever possible dual therapy should be given, at least for a short initial period to all patients with multibacilliferous leprosy. Rifampycin or clofazimine are the drugs usually preferred for giving in combination with dapsone for this purpose, but in theory any drug with antileprotic action that does not belong to the sulphone-sulphonamide group should help to reduce the chance of dapsone resistance.

(3) A good laboratory backing is an essential part of every leprosy treatment programme. Even if full laboratory facilities are not available it is important that good quality skin smear taking and staining and reading must be developed and maintained, especially if resistance is to be realistically tackled. As the writer has travelled in Asia she has seen many centres where the laboratory results cannot be relied upon. Poor results are worse than useless. They produce false negatives more often than false positives and do not give consistent results. It is very easy to take a smear that is too small, to understain or over-decolourize so that when it is examined under the microscope it is impossible to see any acid-fast material. It is also common to find dirty equipment that eliminates the possibility of making good clean slides. It is not difficult to teach a technician how to make a good 
clear well-stained slide, but the doctor or senior paramedical worker needs to be on the alert to keep the standard up. The technician must also have enough time to examine adequately the slide, especially when Morphological Index is required or the acid-fast material is becoming scanty. A well motivated technician does not need to be trained in all aspects of laboratory work to "do" good skin smears. The clinical diagnosis of testing for dapsone resistance depends on good laboratory support. A good leprosy programme cannot exist without a reliable laboratory backing. It is essential that every doctor in leprosy acquaints himself with the laboratory side of the work and takes time to train good technicians (if he cannot get them trained) and to check on the quality of their work from time to time.

(4) Antileprotic therapy should not be stopped for intercurrent diseases or ENL or lepra reaction, which can usually be controlled by use of supportive drugs. If for some reason it is not possible to give an adequate dose of dapsone it is better to use some secondline antileprotic, if available, such as thiosemicarbasone which will not be needed for long term treatment so that if resistance to that drug does develop it will not be such a problem. Although it is generally accepted that dapsone of itself does not cause ENL the writer is one who feels that dapsone in high dosage may, in some patients, increase the severity of the ENL which at low dose is only of nuisance value and becomes disabling on high dosage. Ideally these patients with severe or chronic ENL should receive clofazimine but there are still many countries where this is not freely available and in these situations it may be possible to treat these patients, as we did for many years in Hong Kong before clofazimine became available, with 2 antileprotics in smaller than usual dosage. In theory the use of the 2 drugs together should eliminate the predisposition to emergence of dapsone-resistant organisms.

(5) The maintenance of adequate records is essential. These must include an adequate description of the lesions when first seen, and of any new lesions as they present. It is not enough to just state "Borderline" leprosy as fashions in classification have changed many times over the years. If the lesions are properly described then the next person can tell if there has been any real change in the lesions. It is easiest to have some sort of chart to fill in but do not assume that a space left blank means "normality"-it may not have been examined. If a careful description is given it is possible for someone many years later to classify and compare the present situation with what it was initially. In some centres there are patients who have relapsed and even developed dapsone resistance because initially they were incorrectly classified on the front cover and over the years they have been treated according to that classification. On review after relapse it is possible from the incomplete notes available to see that some were classified as BT who were really BL. Tragedy could have been prevented by a little more care. Please make good examinations and record the findings. Please record all distributions of dapsone, regularity is important and the chart is the place to keep note of it, not in the head of some worker who may not be available when the information is needed. Please record all skin smear results with the examination records. Yes, a book in the laboratory is helpful to the technician but the real place for results is with the other details about the patient, so anyone reading his notes has access to all the relevant information at once.

(6) Educate the patients to understand more about their disease and the necessity of regular prolonged treatment. 
(7) Educate the public so that patients will come earlier when therapy is easier and before severe ENL or deformity have occurred.

(8) Be thorough with the follow-up of patients who have been under treatment for years. They need regular skin and nasal examination (smears) and the whole skin must be examined in a good light. It is no good asking "have you any new skin lesions?" There are many patients under treatment who have never had a complete examination by a doctor or a paramedical worker. How can a patient tell if he has new lesions on his own buttocks?

Dapsone resistance has crept up on us-we blissfully went on believing that it could not happen until we now have a real problem on our hands. In fact it has been said that the problem of getting leprosy under control now is greater than it was 15 years ago, as we now have dapsone resistance. The widespread scattering of dapsone that is taken irregularly is never going to control this disease, especially as now there are patients who will not respond at all to dapsone. We must be more methodical and we must remember that clofazimine is the only drug that we have at present that can be given for long-term treatment of the dapsone-resistant patient. Theoretically resistance to clofazimine could also occur though it has not yet been proven, but we must remember this in the use of clofazimine. The search for new and more effective drugs continues but until and even when another drug becomes available we must remember the lessons of dapsone resistance and use clofazimine as best we know how in the hope that clinical resistance to clofazimine does not occur also. 